The higher rate of positive findings in X-rays requested by our SHOs suggests better judgement based on examination. Perhaps emphasis should be placed on this aspect of care in accident and emergency departments and de-emphasize reliance on radiological diagnosis.

PETER BURDETT-SMITH

Accident and Emergency Department,

The Royal Victoria Infirmary,

Newcastle-upon-Tyne, England

\title{
Computer-unaided diagnosis of acute abdominal pain in an accident and emergency department
}

\section{Sir}

I read with interest the article (Archives of Emergency Medicine [1988] 5, 74-78) by Maitra et al. on computer-unaided diagnosis of acute abdominal pain in an accident and emergency department.

The authors surveyed 200 patients with acute abdominal pain, where the diagnosis was made without computer aid or structured data sheet in the accident and emergency department. The authors claim $65 \%$ to have been correctly diagnosed, claim a normal laparotomy rate of $5 \%$, and conclude that the results compare favourably with those obtained using computer assistance and structured history taking (Adam et al., 1986).

Unfortunately, there are some problems in comparing the results of this presentation with those of the earlier experience which they quote. For example, the authors claim a normal laparotomy rate of $5 \%$ and a negative laparotomy rate of $10.5 \%$; however, in the earlier studies to which they refer, negative laparotomy was defined as the proportion of non-specific patients coming to operation. In the present series, there were only 29 such patients and 10 came to operation (34.5\% - slightly different from the figures quoted, and somewhat higher than the baseline data from earlier trials (Adam et al., 1986).

The diagnostic accuracy quoted $(65 \%)$ is certainly higher than the baseline value in the multi-centre trial (45\%). However, the diagnostic categories chosen by Maitra et al. (1988) are different and somewhat vague. For example, many diagnostic errors concern specific gynaecological diseases; whereas in Maitra's study 'gynaecological' and 'urological' are recorded as disease categories.

One might argue that such a category at least determines the disposal of the patient from the accident and emergency department; but in this connection what is one to make of a category entitled 'peptic perforation, acute peptic ulcer with or without bleeding'.

Even so, only $46 \cdot 7 \%$ of these patients were correctly diagnosed (and only $57 \cdot 7 \%$ of the appendicitis cases), whereas the bad surgical error rate (failure by the initial diagnosis to identify a disease requiring urgent operation) is quoted as $2 \cdot 1 \%$.

It seems curious to say the least that a situation where only about half of the surgical cases are correctly diagnosed can give rise to a bad surgical error rate of $2 \cdot 1 \%$.

Perhaps the problem which has arisen is rooted in the different nature of the studies concerned. The study of Adams et al. (1986) was conducted on a prospective basis; 
whereas this study was retrospective. In this connection it is impossible to agree that the retrospective nature of the study is advantageous, in that as the authors quote it is likely to record the true state of clinical practice'.

The scientific world rightly demands that any innovation (whether computer-based or not), should be subjected to rigorous evaluation; and the authors are to be warmly commended for their scepticism concerning an important innovation. But it seems only reasonable to suggest that studies of the type outlined by Maitra et al. (1988) should be subjected to the same rigour.

F. T. DE DOMBAL

Clinical Information Science Unit, The University of Leeds,

Leeds, England

\section{REFERENCE}

Adam I. D., Chan M., Clifford P. C. et al. (1986) Computer-aided diagnosis of abdominal pain: a multi-centre study. British Medical fournal 293, 800-4.

\section{The use of breath alcohol analysers for diagnosis in the accident and emergency department}

Sir

The use of breath alcohol analysers for diagnosis in the accident and emergency department appears to be well established (Welch et al., 1977; Holt et al., 1980). We are concerned about the lack of evidence for their use in elderly patients.

This department has recently attempted to examine 71 such patients (age range $65-$ 97, mean age 76 years) who presented after a fall. Less than half of them (25) were able to activate the automatic sampling device of the Lion SD2 alcolmeter. There was no difference in the mean ages in the group who were able to use the machine and those who were not. In order to obtain the necessary alveolar air, this automatic device traps a sample after the subject has blown at a minimum flow rate of 28 litres per minute for $2.3 \mathrm{~s}$. Thus a minimum of 1.251 of air is discarded. We found that most of our subjects were unable to maintain this flow rate for long enough, which probably reflects their low tidal volume (Fowler, 1985). It may also reflect failure to understand the instructions or simply lack of co-operation.

Whilst we are embarking on a study to examine this more closely we urge your ON readers to exercise caution in the interpretation of readings taken from elderly patients using this machine.

G. F. TURNER,* G. WARD \& P. WILSON

Department of Geriatric* and Accident and Emergency Medicine, East Birmingham Hospital, Birmingham, England 